http://garmian.edu.krd

https://doi.org/10.24271/garmian.152

\title{
Interaction Effect of Seed Size, Growing Media and Period of Sampling on Growth Characters of Pinus brutia Ten. Seedling at Grdarasha /Erbil
}

\author{
*Shaima A. Karim \\ *Talat M. Ameen \\ *Narin S. Ali \\ * Salahaddin University, Agriculture College, Forestry Department, Erbil, Iraq
}

\begin{abstract}
This investigation was conducted during (25/9/2014 to 25/9/2015) at Grdarasha field/ Salahaddin University, Erbil/College of Agriculture with GPS reading of elevation $419 \mathrm{~m}$. Longitude $36.109 \mathrm{~N}$ Latitude $44.018 \mathrm{E}$. The interaction of seed size, growing media and period of sampling on growth character of Pinus brutia
\end{abstract} Ten. seedling was investigated by using poly bag pots. The studied factors included three seed sizes (large, medium and small), five growth media (control (field soil), control+ loamy soil, control+ compost, control+ peat moss, control+ loamy soil + compost+ peat moss) and three periods (25th of March, 25th of June and September, 2015 ) and their interactions on germination and seedling performance of Pinus brutia Ten. using factorial experiment based on CRD design with three replications. The results indicated that the seed size affected significantly on the studied characters. The highest values of shoot diameter, shoot fresh weight, root fresh weight, shoot dry weight, root dry weight, and Quality index were (2.74, $2.69,1.73,0.94,0.54$, and 0.25 ) recorded from large seed size respectively. While in general the period of sampling affected significantly on the seedling characters (shoot diameter, shoot length, root length, shoot fresh weight, root fresh weight, shoot dry weight, root dry weight, and Quality index). The highest values of them were $(3.13,17.18,68.93,4.16,1.98,1.49,0.76$, and 0.29$)$ recorded from third period respectively.

On the other hand, the growth media affected significantly on most of the studied seedling characters. The highest values of (shoot diameter, shoot fresh weight, root fresh weight, shoot dry weight, and root dry weight) were $(3.12,16.83,4.69,2.26$, 1.55 , and 0.74) recorded from media 3 respectively. Combination treatments among seed size, growth media and period were also affected significantly on most of the studied characters. The highest values of (shoot diameter, shoot length, shoot fresh weight, root fresh weight, shoot dry weight, and root dry weight) were (4.11, $26.50,10.71,4.25,3.88$, and 1.84) which recorded from combination treatment of third period, large seed size and media 3 respectively.

\section{Key words: Growth media, Seed size, Period of sampling, Pine seedlings}




\section{INTRODUCTION}

Pine trees (Pinus brutia Ten.) which belong to pinaceae family distributed as a natural trees in Kurdistan region at Grdarasha/Erbil governorate. They are a medium to large sized evergreen coniferous trees. It grows on most of soils except compact clayey soil (Ali, 2015).

Seed size is an important characteristic of seed quality because larger seeds with larger volume contain more resources and are likely to exhibit greater vigor than smaller seeds (Ellis, 1992).Also Parker et al.( 2004) referred that seed size is an important plant trait that strongly influences the dispersal, establishment, and survival of plant offspring, differences in seed mass among species may span several orders of magnitude within a plant community, with species that have a higher maternal investment in seed tissues generally producing fewer seeds.

The role of seed size in seedling performance has received considerable attention (Foster, 1986; Mazer, 1989; Seiwa and Kikuzawa, 1991; Himanen et al., 2016). Seed sizes in individual lines or cultivars may affect other agronomic characters, different seed size accompany with various levels of starch in each cultivar might be as one factor which influences the expression of physiological dependent character, the seed size is a considerable and significant factor in the germination and early stage of plant growth (Indira et al.,2000).Producing vigorous seedlings in nursery and having information about suitable factors related to seed sowing are essential (Tabatabaii and Ghasriani 1993).

Cicek and Tilki (2007) explained the effects of three seed size (small $>5 \mathrm{~g}$, medium 5-8g and large $<8 \mathrm{~g}$ ) on seed germination, and growth seedling of Castanea sativa. The results indicated that germination parameters were significantly related to seed weight and large seeds germinated early and showed better germination than small seeds.Thus, it can be concluded that large seeds in Castanea sativa have better germination and survival, and the larger the seed, the larger the average seedling would be in seedling diameter, height and dry weight. Soil types are important factors in producing successful seedlings because of their different properties could affect survival and growth (Thompson, 1984). Soil fertility strongly affects foliage growth, for instance nitrogen concentration in soil tends to increase the allocation of carbon to foliage and stem that makes a species a better competitor for light (Vilela and Ravetta, 2001). A convenient growing media should not only supply physical,chemical and biological characteristics required by plants but also provide the conditions for practical plant production (e.g easy to supply, suitable cost, easy processing, lightness and homogenous plant production) (Mathur and Voisin 1996; Sahin and Anapali, 1997).

Growing media is a factor that affects seedling emergence that is used for the germination process. Soil mixtures like soil/sand are preferred instead of single materials for the germination process, because they usually have better physical and chemical characteristics that increase germination percentage in comparison to 
single materials (Aldrete and Mexal, 2005).Furthermore, growing media are materials, other than soils in situ, in which plants are grown. These can include organic materials such as peat, compost, tree bark, coconut (Cocos nucifera L.) coir, poultry feathers, or inorganic materials such as clay, perlite, vermiculite, and mineral wool (Olle and Akvile, 2013).

Ahmadloo et al. (2012) studied the effects of four different soil treatments, including: T1) nursery common soil (control), T2) control soil: cattle manure (5:1), T3) control soil: decomposed litter (5:1), T4) control soil: cattle manure: decomposed litter $(5: 1: 1)$ on seed germination and performance of needle-leaved arizona cypress (Cupressus arizonica var arizonica Greene) and medite cypress (Cupressus sempervirens var. horizantalis Mill.) seedlings in a forest nursery. The results after one year showed that seedlings of both species grown on T4 obtained better germination percent, survival, shoot height, collar diameter, seedling vigor index and Quality Index (QI). Greatest relative growth rate (RGR) of height and diameter were achieved on organic matter treatments. Aklibasinda et al., (2011) determined the effects of different growing media on Scotch pine (Pinus sylvestris) seedling growth attributes such as collar diameter, seedling length, root length, the number of roots, fresh and dry root weight and fresh and dry seedling weight. Eighteen different media were experimented by using rice hulls, pumice and peat materials alone or mixing two of them. The results indicated that growing media had significant effects on plant production parameters. The highest seedling growth attributes were obtained from the media prepared by adding $10 \%$ rice hulls to peat material. Aeration porosity and usable water amount as volume of this media was $60.1 \%$ and $15.9 \%$, respectively. Among the pure media, it was found that seedling could not grow in pure rice hulls media. Seedling production process in forest nursery mostly depend on the seed that is why choosing good seed has a great role in producing sound seedling and making processes easier and faster also will be more resist to environmental difficulties, so the aim of this study was investigating the effect of seed size, growing media and period of sampling on growth characters of Pinus brutia ten. seedling.

\section{MATERIALS AND METHODS}

An experiment was carried out at Grdarasha field / Salahaddin University, Erbil during 25/9/ 2014 until 25/9/2015. The field is far away from the center of Erbil government by $(5 \mathrm{~km})$ located at elevation $419 \mathrm{~m}$. Longitude $36.109 \mathrm{~N}$ Latitude 44.018 E .The experiment was arranged out as factorial, based on CRD design with three replications. Three different seed size were used in which large seeds ranging between $(5.1-6.6 .3 \mathrm{~mm})$, medium seeds ranging between $(3.9-4.9 \mathrm{~mm})$ and small seed size (3.4-4.1 mm), five growing media also were tested which include field soil with a silty clay loam textural represented as a (control), control+ loamy soil, control+ compost, control+ peat moss, control+ loamy soil + compost+ peat moss 
$(1,1: 1,1: 1,1: 1,1: 1: 1: 1)$ by volume respectively, a total of 300 polybags had been depended for all the experiment.

The growing soil media was analyzed at the laboratory of research center/ Agriculture College, Salahaddin University / Erbil/ Iraq (Table 1) .The seeds were sank in water for $24 \mathrm{hr}$.to speed the germination, and five seeds were sown in a polybags on 25/9/2014; seeds germination began in the 13 of October in all the soil treatments, and the germination was recorded every 3 days for 37 days. Germination percent was determined by using the following equation:

Germination $\%=\frac{\text { Numbero f seedsinitiated }}{\text { numbero fgerminating seeds }} * 100 \quad$ (Ismail and Kardoush, 2011)

Table (1) Chemical characteristics of different soil combinations

\begin{tabular}{|c|c|c|c|c|c|}
\hline Elements & $\begin{array}{c}\text { field soil } \\
\text { (control) }\end{array}$ & $\begin{array}{c}\text { Control } \\
+ \text { Loamy } \\
\text { soil }\end{array}$ & $\begin{array}{c}\text { Control+ } \\
\text { compost }\end{array}$ & $\begin{array}{c}\text { Control+ } \\
\text { peat moss }\end{array}$ & $\begin{array}{c}\text { Control+loamy } \\
\text { soil+composite+ } \\
\text { peat moss }\end{array}$ \\
\hline $\begin{array}{c}\text { PH (at } \\
\left.16 \mathrm{c}^{\circ}\right)\end{array}$ & 7.42 & 6.90 & 6.94 & 6.0 & 6.95 \\
\hline $\begin{array}{c}\mathrm{EC}(\mathrm{Dsm}- \\
1)\end{array}$ & 0.2 & 1.55 & 6.53 & 4.43 & 5.20 \\
\hline $\mathrm{N} \%$ & 0.18 & 0.06 & 0.07 & 0.07 & 0.08 \\
\hline $\mathrm{P}(\mathrm{ppm})$ & 3.75 & 4.5 & 4.2 & 4.7 & 5.2 \\
\hline $\mathrm{K}(\mathrm{ppm})$ & 78 & 41 & 51 & 39 & 37 \\
\hline $\mathrm{Ca}(\mathrm{ppm})$ & 60 & 117 & 123 & 124 & 74 \\
\hline
\end{tabular}

Initiate and complete the germination of seed varied in different seed size , the minimum emergence time taken was (23-26) days by small seed size, (22-24) days by medium seed size and (81-21) days by large seed size. The germination percentage significantly declined with reduction in seed size which were (87.973, 79.527 and 71.973) for large, medium and small seed size respectively.

After completion of field germination only one seedling (best one) per polybag was maintained to record the initial growth parameters. Proper cares, including artificial watering, weed removal each 10 day (manual), and root pruning of seedlings (twice during the growth period) were carried out regularly, three seedlings were randomly selected in each combination of treatment (seed sizegrowing media). The growth parameters were measured at three period (25 March, 25 June and 25 September) which included shoot height, root length, were defined as the distance from the top of the root plug to the upper and lower end of a seedling, respectively and the shoot diameter were measured using a digital caliper; after separating root system and shoot (stem + needle), root dry weight and shoot dry weight were assessed after oven-drying at $70{ }^{\circ} \mathrm{C}$ for $48 \mathrm{hr}$.

Seedling quality index (QI) was calculated by using below formula:

The seedling quality index $(\mathrm{QI})=\frac{\text { Total seedling dry weight }}{\mathrm{r}}$

(Ahmadloo et al. ,2012) 


\section{Data analysis:}

Data was statistically analyzed using SPSS program (version 20), to determination the interaction effect of seeds size, growing media and period treatments on germination, survival, growth indices depending on paired and nonpaired t-test. (Al-Rawi and Khalaf Allah, 2000).

\section{RESULTS AND DISCUSSION}

Generally seedling derived from seeds that were of large size have been significantly larger than seedlings derived from medium and small seed size; which were affected significantly at level of significance 0.01 on most of the studied characters except the shoot length and root length which were not affected significantly by seed sizes. The highest values of (shoot diameter, shoot fresh weight, root fresh weight, shoot dry weight, root dry weight, Quality index ) were $(2.74,2.69,1.73,0.94,0.54$, and 0.25$)$ recorded from large seed size respectively, while the lowest values of them were $(2.29,1.86,1.14,0.59,0.34$ and 0.16$)$ obtained from small seed size as it's shown in (Table2). The observed results with regard to seed germination are in concurrence with increase in germination, seed size and weight have been reported in tree species like Hardwickia binata (Sharma and Sood , 1990) ) and Abies pindrow (Singh and Shah, 1992) similar results have been observed in Pongamia pinnata seed germination (Arjunan et al.,1994). Heavy and large seed contains more food reserves than smaller ones, which is helpful in germination by providing more energy (Lusk, 1995), and our results made clear that, the speed of germination in large seed size was faster than other seed size, some authors have hypothesized that a large seed enables a seedling to allocate proportionally more resources to root development than does a smaller seed. Similar results were obtained by (Salisbury, 1974 ) and (Cicek and Tilki, 2007).

Table (2) Effect of seed size on some characteristics of Pinus brutia Ten. seedling

\begin{tabular}{|c|c|c|c|c|c|c|c|c|}
\hline $\begin{array}{c}\text { See } \\
\mathrm{d} \\
\text { size } \\
\text { diam } \\
\text { eter } \\
(\mathrm{mm})\end{array}$ & $\begin{array}{c}\text { shoot } \\
\text { dength }\end{array}$ & $\begin{array}{c}\text { shoot } \\
\text { length }\end{array}$ & $\begin{array}{c}\text { shoot } \\
\text { fresh } \\
\text { weight }\end{array}$ & $\begin{array}{c}\text { root } \\
\text { fresh } \\
\text { weight }\end{array}$ & $\begin{array}{c}\text { shoot } \\
\text { dry } \\
\text { weigh } \\
\mathrm{t}\end{array}$ & $\begin{array}{c}\text { root } \\
\text { dry } \\
\text { weig } \\
\mathrm{ht}\end{array}$ & $\begin{array}{c}\text { Quality } \\
\text { index }\end{array}$ \\
\cline { 2 - 8 } & \multicolumn{2}{|c|}{$(\mathrm{cm})$} & \multicolumn{5}{|c|}{$(\mathrm{g})$} \\
\hline $\mathrm{L}$ & $2.74^{\mathrm{a}}$ & $12.92^{\mathrm{a}}$ & $53.64^{\mathrm{a}}$ & $2.69^{\mathrm{a}}$ & $1.73^{\mathrm{a}}$ & $0.94^{\mathrm{a}}$ & $0.54^{\mathrm{a}}$ & $0.25^{\mathrm{a}}$ \\
\hline $\mathrm{M}$ & $2.56^{\mathrm{a}}$ & $12.44^{\mathrm{a}}$ & $60.66^{\mathrm{a}}$ & $2.37^{\mathrm{ab}}$ & $1.49^{\mathrm{ab}}$ & $0.76^{\mathrm{ab}}$ & $0.45^{\mathrm{a}}$ & $0.21^{\mathrm{ab}}$ \\
\hline $\mathrm{S}$ & $2.29^{\mathrm{b}}$ & $11.67^{\mathrm{a}}$ & $53.53^{\mathrm{a}}$ & $1.86^{\mathrm{b}}$ & $1.14^{\mathrm{b}}$ & $0.59^{\mathrm{b}}$ & $0.34^{\mathrm{b}}$ & $0.16^{\mathrm{b}}$ \\
\hline
\end{tabular}


Table (3) Effect of different growth media on some characteristics of Pinus brutia Ten. seedling.

\begin{tabular}{|c|l|l|l|l|l|l|l|l|}
\hline $\begin{array}{c}\text { Growth } \\
\text { media }\end{array}$ & $\begin{array}{c}\text { shoot } \\
\text { diameter } \\
(\mathrm{mm})\end{array}$ & $\begin{array}{c}\text { shoot } \\
\text { length } \\
(\mathrm{cm})\end{array}$ & $\begin{array}{c}\text { root } \\
\text { length } \\
(\mathrm{cm})\end{array}$ & $\begin{array}{c}\text { shoot } \\
\text { fresh } \\
\text { weight }\end{array}$ & $\begin{array}{c}\text { root } \\
\text { fresh } \\
\text { weight }\end{array}$ & $\begin{array}{c}\text { shoot } \\
\text { dry } \\
\text { weight }\end{array}$ & $\begin{array}{c}\text { root } \\
\text { dry } \\
\text { weight }\end{array}$ & $\begin{array}{c}\text { Quality } \\
\text { index }\end{array}$ \\
\cline { 3 - 9 } & \multicolumn{2}{|c|}{$(\mathrm{cm})$} & \multicolumn{6}{|c|}{$(\mathrm{g})$} \\
\hline M1 & $2.32^{\mathrm{b}}$ & $8.69^{\mathrm{c}}$ & $53.60^{\mathrm{a}}$ & $1.03^{\mathrm{c}}$ & $0.85^{\mathrm{c}}$ & $0.35^{\mathrm{c}}$ & $0.28^{\mathrm{c}}$ & $0.30^{\mathrm{a}}$ \\
\hline M2 & $2.27^{\mathrm{b}}$ & $8.62^{\mathrm{c}}$ & $52^{\mathrm{a}}$ & $0.89^{\mathrm{c}}$ & $0.94^{\mathrm{c}}$ & $0.30^{\mathrm{c}}$ & $0.26^{\mathrm{c}}$ & $0.18^{\mathrm{b}}$ \\
\hline M3 & $3.12^{\mathrm{a}}$ & $16.83^{\mathrm{a}}$ & $51.67^{\mathrm{a}}$ & $4.69^{\mathrm{a}}$ & $2.26^{\mathrm{a}}$ & $1.55^{\mathrm{a}}$ & $0.74^{\mathrm{a}}$ & $0.29^{\mathrm{a}}$ \\
\hline M4 & $2.41^{\mathrm{b}}$ & $11.60^{\mathrm{b}}$ & $62.41^{\mathrm{a}}$ & $1.66^{\mathrm{c}}$ & $1.47^{\mathrm{b}}$ & $0.59^{\mathrm{c}}$ & $0.40^{\mathrm{bc}}$ & $0.15^{\mathrm{b}}$ \\
\hline M5 & $2.53^{\mathrm{b}}$ & $15.97^{\mathrm{a}}$ & $60.04^{\mathrm{a}}$ & $3.27^{\mathrm{b}}$ & $1.75^{\mathrm{b}}$ & $1.03^{\mathrm{b}}$ & $0.54^{\mathrm{b}}$ & $0.19^{\mathrm{b}}$ \\
\hline
\end{tabular}

$* \mathrm{M} 5=$ control + sandy loam + compost + peat moss

Table (3) explained that the growth media were affected significantly at level of significance 0.01 on most of the studied characters except root length which was not affected significantly by media. The highest values of (shoot diameter, shoot fresh weight, root fresh weight, shoot dry weight, and root dry weight) were $(3.12,16.83,4.69,2.26,1.55$, and 0.74$)$ recorded from media (3) respectively, and the lowest values of them were $(2.27,8.62,0.89,0.30$, and 0.26$)$ obtained from media(2) respectively. While the lowest value of root fresh weight was (0.85) recorded from media (1).

On the other hand the highest and lowest values of quality index (0.30 and 0.15$)$ were recorded from growth media ( 1 and 4 ) respectively, this implies that the plant experiences high development, and this may be due to nutritional elements (Table 1) in media (3) lead to improvement growth indices. Similar instances were also found by Durgapal et al. (2002) whereas combination of soil organic matter improved the seed germination and subsequent growth of Cedrus deodara and Pinus wallichiana under nursery conditions, and in Ahmadloo, et al. (2012) whereas the seedlings of Cupressus arizonica and Cupressus. sempervirens var. horizantalis (Mill.) obtained better germination percent, survival, shoot height, collar diameter, seedling vigor index and Quality Index (QI), seedlings grown on soil mixture include control soil: cattle manure: decomposed litter, on the other hand the highest germination percentage resulted in cow-dung mixture soil medium might be due to it helps to develop and maintain good soil structure and porosity, water holding capacity, aeration, permeability and contribute in raising Cation Exchange Capacity (CEC) of the soil. 
Table (4) showed that in general the periods were affected significantly at level of significance 0.01 on the studied characteristics of seedlings (shoot diameter, shoot length, root length, shoot fresh weight, root fresh weight, shoot dry weight, root dry weight, and Quality index). The highest values of them were $(3.13,17.18$, $68.93,4.16,1.98,1.49,0.76$, and 0.29 ) recorded from third period respectively, while the lowest values of them were $(1.77,7.19,39.25,0.76,0.94,0.19,0.15$, and 0.06) obtained from first period. This may be due to temperature, light density, period lighting, and environmental characters which were differing among periods.

Table (4) Effect of periods on some characteristics of Pinus brutia Ten. seedlings

\begin{tabular}{|c|c|c|c|c|c|c|c|c|}
\hline Period & $\begin{array}{c}\text { shoot } \\
\text { diameter } \\
(\mathrm{mm})\end{array}$ & $\begin{array}{c}\text { shoot } \\
\text { length }\end{array}$ & $\begin{array}{c}\text { root } \\
\text { length }\end{array}$ & $\begin{array}{c}\text { shoot } \\
\text { fresh } \\
\text { weight }\end{array}$ & $\begin{array}{c}\text { root } \\
\text { fresh } \\
\text { weight }\end{array}$ & $\begin{array}{c}\text { shoot } \\
\text { dry } \\
\text { weight }\end{array}$ & $\begin{array}{c}\text { root } \\
\text { dry } \\
\text { weight }\end{array}$ & $\begin{array}{c}\text { Quality } \\
\text { index }\end{array}$ \\
\cline { 2 - 8 } & \multicolumn{2}{|c|}{$(\mathrm{cm})$} & \multicolumn{5}{|c|}{$(\mathrm{g})$} & \\
\hline First & $1.77^{\mathrm{c}}$ & $7.19^{\mathrm{c}}$ & $39.25^{\mathrm{c}}$ & $0.76^{\mathrm{c}}$ & $0.94^{\mathrm{c}}$ & $0.19^{\mathrm{c}}$ & $0.15^{\mathrm{c}}$ & $0.06^{\mathrm{b}}$ \\
\hline Second & $2.69^{\mathrm{b}}$ & $12.66^{\mathrm{b}}$ & $59.64^{\mathrm{b}}$ & $2.00^{\mathrm{b}}$ & $1.44^{\mathrm{b}}$ & $0.61^{\mathrm{b}}$ & $0.43^{\mathrm{b}}$ & $0.27^{\mathrm{a}}$ \\
\hline Third & $3.13^{\mathrm{a}}$ & $17.18^{\mathrm{a}}$ & $68.93^{\mathrm{a}}$ & $4.16^{\mathrm{a}}$ & $1.98^{\mathrm{a}}$ & $1.49^{\mathrm{a}}$ & $0.76^{\mathrm{a}}$ & $0.29^{\mathrm{a}}$ \\
\hline
\end{tabular}

Table (5) showed that the interaction between (seed size and growth media) affected significantly at level of significance 0.01 on most of the studied characters except root length was not affected significantly by combination treatments. The highest values were $(3.45,17.88,5.50,2.67,1.93,0.93$, and 0.37$)$ of (shoot diameter ,shoot length, shoot fresh weight, root fresh weight, shoot dry weight, root dry weight, and quality index) recorded from large seed size under media(3) respectively, while the lowest values were $(7.42,0.62,0.60$, and 0.18 ) of (shoot length, shoot fresh weight, root fresh weight, root dry weight) obtained from small seed size under media(1) respectively. On the other hand the lowest values were $(2.03,0.23$, and 0.08$)$ of (shoot diameter, shoot dry weight, and quality index) recorded from small seed size under media (2) respectively. There interaction created different condition for plant growth which was affected on the studied characters (Darwesh, 2007). 
Table (5) interaction effect of seed size and different growing media on characteristics of Pinus brutia Ten.seedling.

\begin{tabular}{|c|c|c|c|c|c|c|c|c|c|}
\hline \multirow[t]{2}{*}{$\begin{array}{c}\text { Seed } \\
\text { size }\end{array}$} & \multirow[t]{2}{*}{ Media } & \multirow[t]{2}{*}{$\begin{array}{c}\text { shoot } \\
\text { diameter } \\
(\mathrm{mm})\end{array}$} & $\begin{array}{l}\text { shoot } \\
\text { length }\end{array}$ & $\begin{array}{l}\text { root } \\
\text { length }\end{array}$ & $\begin{array}{c}\text { shoot } \\
\text { fresh } \\
\text { weight }\end{array}$ & $\begin{array}{c}\text { root } \\
\text { fresh } \\
\text { weight }\end{array}$ & $\begin{array}{c}\text { shoot } \\
\text { dry } \\
\text { weight }\end{array}$ & $\begin{array}{c}\text { root } \\
\text { dry } \\
\text { weight }\end{array}$ & \multirow[t]{2}{*}{$\begin{array}{l}\text { Quality } \\
\text { index }\end{array}$} \\
\hline & & & \multicolumn{2}{|c|}{$(\mathrm{cm})$} & \multicolumn{4}{|c|}{ (g) } & \\
\hline \multirow{5}{*}{$\mathrm{L}$} & M1 & $2.55^{\mathrm{abc}}$ & $9.44^{\mathrm{b}-\mathrm{e}}$ & $44.13^{\mathrm{a}}$ & $1.25^{\mathrm{cd}}$ & $0.93^{\mathrm{cd}}$ & $0.36^{\mathrm{bc}}$ & $0.32^{\mathrm{b}}$ & $0.33^{\mathrm{ab}}$ \\
\hline & M2 & $2.37^{b c}$ & $9.61^{b-e}$ & $54.29^{a}$ & $1.04^{\mathrm{cd}}$ & $1.16^{\mathrm{bcd}}$ & $0.38^{\mathrm{bc}}$ & $0.29^{\mathrm{b}}$ & $0.14^{\mathrm{ab}}$ \\
\hline & M3 & $3.45^{\mathrm{a}}$ & $17.88^{\mathrm{a}}$ & $46.67^{a}$ & $5.50^{\mathrm{a}}$ & $2.67^{\mathrm{a}}$ & $1.93^{\mathrm{a}}$ & $0.93^{\mathrm{a}}$ & $0.37^{\mathrm{a}}$ \\
\hline & M4 & $2.54^{\mathrm{abc}}$ & $12.05^{\mathrm{a}-\mathrm{e}}$ & $59.44^{\mathrm{a}}$ & $1.93^{\mathrm{bcd}}$ & $1.69^{\mathrm{a}-\mathrm{d}}$ & $0.80^{\mathrm{bc}}$ & $0.51^{\mathrm{ab}}$ & $0.19^{\mathrm{ab}}$ \\
\hline & M5 & $2.80^{b c}$ & $15.61^{\mathrm{a}-\mathrm{d}}$ & $63.67^{\mathrm{a}}$ & $3.76^{\mathrm{a}-\mathrm{d}}$ & $2.19^{\mathrm{ab}}$ & $1.22^{\mathrm{abc}}$ & $0.67^{\mathrm{ab}}$ & $0.24^{\mathrm{ab}}$ \\
\hline \multirow{5}{*}{ M } & M1 & $2.31^{b c}$ & $9.21^{\mathrm{b}-\mathrm{e}}$ & $64.89^{\mathrm{a}}$ & $1.21^{\mathrm{cd}}$ & $1.01^{\mathrm{bcd}}$ & $0.44^{\mathrm{bc}}$ & $0.34^{\mathrm{b}}$ & $0.32^{\mathrm{ab}}$ \\
\hline & M2 & $2.41^{b c}$ & $8.40^{\text {cde }}$ & $54.09^{a}$ & $0.90^{\text {cd }}$ & $0.89^{\mathrm{cd}}$ & $0.29^{\mathrm{bc}}$ & $0.30^{b}$ & $0.13^{\mathrm{ab}}$ \\
\hline & M3 & $3.14^{\mathrm{ab}}$ & $16.57^{\mathrm{ab}}$ & $53.56^{\mathrm{a}}$ & $4.65^{\mathrm{ab}}$ & $2.16^{\mathrm{ab}}$ & $1.47^{\mathrm{ab}}$ & $0.67^{\mathrm{ab}}$ & $0.26^{\mathrm{ab}}$ \\
\hline & M4 & $2.33^{b c}$ & $11.22^{\mathrm{a}-\mathrm{e}}$ & $65.56^{\mathrm{a}}$ & $1.52^{\mathrm{cd}}$ & $1.46^{\mathrm{bcd}}$ & $0.50^{\mathrm{bc}}$ & $0.38^{b}$ & $0.14^{\mathrm{ab}}$ \\
\hline & M5 & $2.60^{\mathrm{abc}}$ & $16.80^{\mathrm{ab}}$ & $65.22^{a}$ & $3.56^{\mathrm{a}-\mathrm{d}}$ & $1.95^{\mathrm{abc}}$ & $1.11^{\mathrm{abc}}$ & $0.58^{\mathrm{ab}}$ & $0.20^{\mathrm{ab}}$ \\
\hline \multirow{5}{*}{ S } & M1 & $2.11^{b c}$ & $7.42^{\mathrm{e}}$ & $51.78^{\mathrm{a}}$ & $0.62^{\mathrm{d}}$ & $0.60^{\mathrm{d}}$ & $0.26^{\mathrm{c}}$ & $0.18^{b}$ & $0.24^{\mathrm{ab}}$ \\
\hline & M2 & $2.03^{\mathrm{c}}$ & $7.84^{\mathrm{de}}$ & $47.63^{\mathrm{a}}$ & $0.74^{\text {cd }}$ & $0.78^{\mathrm{cd}}$ & $0.23^{\mathrm{c}}$ & $0.20^{b}$ & $0.08^{b}$ \\
\hline & M3 & $2.77^{\mathrm{abc}}$ & $16.04^{\mathrm{abc}}$ & $54.78^{\mathrm{a}}$ & $3.93^{\mathrm{abc}}$ & $1.96^{\mathrm{abc}}$ & $1.23^{\mathrm{abc}}$ & $0.61^{\mathrm{ab}}$ & $0.23^{\mathrm{ab}}$ \\
\hline & M4 & $2.35^{b c}$ & $11.53^{\mathrm{a}-\mathrm{e}}$ & $62.22^{\mathrm{a}}$ & $1.53^{\mathrm{cd}}$ & $1.26^{\mathrm{bcd}}$ & $0.47^{b c}$ & $0.33^{b}$ & $0.12^{\mathrm{ab}}$ \\
\hline & M5 & $2.19^{b c}$ & $15.51^{\mathrm{a}-\mathrm{d}}$ & $51.22^{\mathrm{a}}$ & $2.48^{\mathrm{a}}$ & $1.12^{\mathrm{bcd}}$ & $0.75^{b c}$ & $0.38^{\mathrm{b}}$ & $0.12^{\mathrm{ab}}$ \\
\hline
\end{tabular}

$* \mathrm{~L}=$ large size $\quad * \mathrm{M}=$ medium size $\quad * \mathrm{~S}=$ small size

$* \mathrm{M} 1=$ Control $* \mathrm{M} 2=$ control + sandy loam $* \mathrm{M} 3=$ control + compost $* \mathrm{M} 4=$ control +peat mos $*$ M5= control +sandy loam+ compost + peat moss

Table (6) Explained that combination treatments (growth media and periods) were affected significantly at level of significance 0.01 on most of the studied characters except root length and quality index which were recorded (76.89 and 0.64) from third and second periods under media (1) respectively and obtained were (33.55 and 0.04) from first periods under media (3 and 5) respectively. The highest values were $(3.79,25.61,8.97,3.36,3.19$, and 1.39)of (shoot diameter, shoot length, shoot fresh weight, root fresh weight, shoot dry weight and root dry weight) recorded from third periods under media (3) respectively, while the lowest values were $(5.95,0.5$, and 0.68 ) of (shoot length, shoot fresh weight, root fresh weight) obtained from first period under media (1) respectively. On the other hand the lowest values were (1.55 and 0.12) of (shoot diameter and shoot dry weight) recorded from first period under media (4) respectively. Similar results obtained by (Ahmadloo et al., 2012). 
Table (6) Interaction effect of different periods and growing media on some characteristics of Pinus brutia Ten. seedlings

\begin{tabular}{|c|c|c|c|c|c|c|c|c|c|}
\hline \multirow[t]{2}{*}{ Period } & \multirow[t]{2}{*}{ Media } & \multirow[t]{2}{*}{$\begin{array}{c}\text { shoot } \\
\text { diameter } \\
(\mathrm{mm})\end{array}$} & $\begin{array}{l}\text { shoot } \\
\text { length }\end{array}$ & $\begin{array}{l}\text { root } \\
\text { length }\end{array}$ & $\begin{array}{c}\text { shoot } \\
\text { fresh } \\
\text { weight }\end{array}$ & $\begin{array}{c}\text { root } \\
\text { fresh } \\
\text { weight }\end{array}$ & $\begin{array}{c}\text { shoot } \\
\text { dry } \\
\text { weight }\end{array}$ & $\begin{array}{c}\text { root } \\
\text { dry } \\
\text { weight }\end{array}$ & \multirow[t]{2}{*}{$\begin{array}{l}\text { Quality } \\
\text { index }\end{array}$} \\
\hline & & & \multicolumn{2}{|c|}{$(\mathrm{cm})$} & \multicolumn{4}{|c|}{ (g) } & \\
\hline \multirow{5}{*}{ First } & M1 & $1.58^{\mathrm{e}}$ & $5.95^{\mathrm{g}}$ & $34.13^{f}$ & $0.5^{f}$ & $0.68^{f}$ & $0.18^{\mathrm{de}}$ & $0.15^{\mathrm{e}}$ & $0.06^{\mathrm{d}}$ \\
\hline & M2 & $1.77^{\mathrm{e}}$ & $6.71^{\mathrm{fg}}$ & $36.01^{\text {ef }}$ & $0.66^{f}$ & $0.79^{\mathrm{ef}}$ & $0.18^{\mathrm{de}}$ & $0.13^{\mathrm{e}}$ & $0.06^{\mathrm{d}}$ \\
\hline & M3 & $2.33^{\mathrm{d}}$ & $8.64^{\mathrm{efg}}$ & $33.55^{\mathrm{f}}$ & $1.16^{\mathrm{ef}}$ & $1.09^{\mathrm{def}}$ & $0.32^{\mathrm{de}}$ & $0.17^{\mathrm{e}}$ & $0.08^{d}$ \\
\hline & M4 & $1.55^{\mathrm{e}}$ & $6.93^{\mathrm{fg}}$ & $51.55^{\mathrm{b}-\mathrm{f}}$ & $0.69^{f}$ & $1.18^{\mathrm{def}}$ & $0.12^{\mathrm{e}}$ & $0.15^{\mathrm{e}}$ & $0.05^{\mathrm{d}}$ \\
\hline & M5 & $1.62^{\mathrm{e}}$ & $7.72^{\text {efg }}$ & $41 \mathrm{~d}^{\mathrm{ef}}$ & $0.74^{\mathrm{f}}$ & $0.96^{\mathrm{def}}$ & $0.15^{\mathrm{e}}$ & $0.12^{\mathrm{e}}$ & $0.04^{\mathrm{d}}$ \\
\hline \multirow{5}{*}{ Second } & M1 & $2.40^{\mathrm{d}}$ & $9.67^{\mathrm{ef}}$ & $49.78^{\mathrm{c}-\mathrm{f}}$ & $0.98^{\mathrm{ef}}$ & $0.82^{\mathrm{ef}}$ & $0.31^{\mathrm{de}}$ & $0.28^{\mathrm{de}}$ & $0.64^{\mathrm{a}}$ \\
\hline & M2 & $2.60^{\mathrm{cd}}$ & $9 \mathrm{ef}^{\mathrm{g}}$ & $61^{a-d}$ & $1.01^{\mathrm{ef}}$ & $1.08^{\mathrm{def}}$ & $0.34^{\mathrm{de}}$ & $0.32^{\mathrm{de}}$ & $0.14^{\mathrm{cd}}$ \\
\hline & M3 & $3.24^{b}$ & $16.23^{b}$ & $57^{\mathrm{a}-\mathrm{e}}$ & $3.95^{\mathrm{c}}$ & $2.33^{b c}$ & $1.13^{\mathrm{c}}$ & $0.65^{\mathrm{c}}$ & $0.26^{b c}$ \\
\hline & M4 & $2.68^{\mathrm{cd}}$ & $13.05^{\mathrm{cd}}$ & $65.44^{\mathrm{abc}}$ & $1.52^{\mathrm{def}}$ & $1.32^{\mathrm{def}}$ & $0.50^{\mathrm{de}}$ & $0.39^{\text {cde }}$ & $0.15^{\mathrm{cd}}$ \\
\hline & M5 & $2.50^{\mathrm{cd}}$ & $15.33^{\mathrm{bc}}$ & $65^{\mathrm{abc}}$ & $2.54^{\text {cde }}$ & $1.67^{\text {cde }}$ & $0.76^{\mathrm{cd}}$ & $0.52^{\text {cd }}$ & $0.18^{\mathrm{cd}}$ \\
\hline \multirow{5}{*}{ Third } & M1 & $2.98^{\mathrm{bc}}$ & $10.45^{\mathrm{de}}$ & $76.89^{\mathrm{a}}$ & $1.55^{\mathrm{def}}$ & $1.04^{\mathrm{def}}$ & $0.57^{\mathrm{de}}$ & $0.40^{\text {cde }}$ & $0.19^{\text {bcd }}$ \\
\hline & M2 & $2.43^{\mathrm{cd}}$ & $10.14^{\mathrm{de}}$ & $59 a^{d}$ & $1.01^{\mathrm{ef}}$ & $0.96^{\mathrm{def}}$ & $0.37^{\mathrm{de}}$ & $0.33^{\mathrm{de}}$ & $0.12^{\mathrm{cd}}$ \\
\hline & M3 & $3.79^{\mathrm{a}}$ & $25.61^{\mathrm{a}}$ & $64.44^{\mathrm{abc}}$ & $8.97^{\mathrm{a}}$ & $3.36^{\mathrm{a}}$ & $3.19^{\mathrm{a}}$ & $1.39^{\mathrm{a}}$ & $0.52^{\mathrm{a}}$ \\
\hline & M4 & $2.99^{b c}$ & $14.82^{\mathrm{bc}}$ & $70.22^{\mathrm{abc}}$ & $2.76^{\mathrm{cd}}$ & $1.90^{\mathrm{bcd}}$ & $1.14^{\mathrm{c}}$ & $0.67^{\mathrm{c}}$ & $0.25^{b c}$ \\
\hline & M5 & $3.48^{\mathrm{ab}}$ & $24.87^{\mathrm{a}}$ & $74.11^{\mathrm{ab}}$ & $6.52^{b}$ & $2.63^{\mathrm{ab}}$ & $2.17^{b}$ & $0.99^{b}$ & $0.34^{b}$ \\
\hline
\end{tabular}

$* \mathrm{M} 1=$ Control $* \mathrm{M} 2=$ control + sandy loam $* \mathrm{M} 3=$ control + compost $* \mathrm{M} 4=$ control +peat moss $*$ M5= control +sandy loam+ compost + peat moss

Table (7) showed that in general combination treatments (seed size and periods) were affected significantly at level of significance 0.01 on the studied characteristics of seedlings. (shoot diameter, shoot length, shoot fresh weight, root fresh weight, shoot dry weight, root dry weight, and Quality index).The highest values of them were $(3.34,17.87,4.87,2.35,1.81,0.93,0.93$, and 0.36$)$ recorded from third periods in large seed size respectively except root length which (87.07) was recorded from third periods in medium seed size, while the lowest values of them were $(1.55,6.47,34.61,0.63,0.66,0.12,0.11$, and 0.04$)$ obtained from first period in small seed size respectively. There interaction created different conditions for plant growth which were affected on the studied characters (Darwesh, 2007). 
Table (7) Interaction effect of seed size and periods on some characteristics of Pinus brutia Ten. seedlings

\begin{tabular}{|c|c|c|c|c|c|c|c|c|c|}
\hline \multirow[t]{2}{*}{$\begin{array}{c}\text { seed } \\
\text { size }\end{array}$} & \multirow[t]{2}{*}{ Period } & $\begin{array}{c}\text { shoot } \\
\text { diameter } \\
(\mathrm{mm})\end{array}$ & $\begin{array}{l}\text { shoot } \\
\text { length }\end{array}$ & $\begin{array}{l}\text { root } \\
\text { length }\end{array}$ & $\begin{array}{c}\text { shoot } \\
\text { fresh } \\
\text { weight }\end{array}$ & $\begin{array}{c}\text { root } \\
\text { fresh } \\
\text { weight }\end{array}$ & $\begin{array}{c}\text { shoot } \\
\text { dry } \\
\text { weight }\end{array}$ & $\begin{array}{c}\text { root } \\
\text { dry } \\
\text { weight }\end{array}$ & \multirow[t]{2}{*}{$\begin{array}{l}\text { Quality } \\
\text { index }\end{array}$} \\
\hline & & & \multicolumn{2}{|c|}{$(\mathrm{cm})$} & \multicolumn{4}{|c|}{ (g) } & \\
\hline \multirow{3}{*}{$\mathrm{L}$} & First & $1.91^{\mathrm{de}}$ & $7.96^{\mathrm{cd}}$ & $41.82^{\mathrm{cd}}$ & $0.88^{d}$ & $1.11^{\mathrm{bc}}$ & $0.28^{\mathrm{c}}$ & $0.18^{d}$ & $0.07^{\mathrm{bc}}$ \\
\hline & Second & $2.97^{\mathrm{ab}}$ & $12.93^{\mathrm{ab}}$ & $57.47^{\mathrm{bc}}$ & $2.33^{\mathrm{bcd}}$ & $1.72^{\mathrm{ab}}$ & $0.72 b c$ & $0.53^{\mathrm{bc}}$ & $0.33^{\mathrm{a}}$ \\
\hline & Third & $3.34^{\mathrm{a}}$ & $17.87^{\mathrm{a}}$ & $61.63^{\mathrm{ab}}$ & $4.87^{\mathrm{a}}$ & $2.35^{\mathrm{a}}$ & $1.81 \mathrm{a}$ & $0.93^{\mathrm{a}}$ & $0.36^{\mathrm{a}}$ \\
\hline \multirow{3}{*}{$\mathrm{M}$} & First & 1.8 & $7.15^{\mathrm{cd}}$ & $41.32^{\mathrm{cd}}$ & $0.76^{d}$ & $1.04^{\mathrm{bc}}$ & $0.18 \mathrm{c}$ & $0.15^{\mathrm{d}}$ & $0.06^{\mathrm{bc}}$ \\
\hline & Second & $2.72^{b c}$ & $13.11^{\mathrm{ab}}$ & $62.60^{\mathrm{ab}}$ & $2.08^{\mathrm{bcd}}$ & $1.45^{\mathrm{abc}}$ & $0.61 b c$ & $0.43^{\mathrm{cd}}$ & $0.27^{\mathrm{a}}$ \\
\hline & Third & $3.12^{\mathrm{ab}}$ & $17.07^{\mathrm{ab}}$ & $87.07^{\mathrm{a}}$ & $4.27^{\mathrm{ab}}$ & $1.99^{\mathrm{ab}}$ & $1.51 \mathrm{a}$ & $0.77^{\mathrm{ab}}$ & $0.30^{\mathrm{a}}$ \\
\hline \multirow{3}{*}{$\mathrm{S}$} & First & $1.55^{\mathrm{e}}$ & $6.47^{\mathrm{d}}$ & $34.61^{d}$ & $0.63^{d}$ & $0.66^{\mathrm{c}}$ & $0.12 \mathrm{c}$ & $0.11^{\mathrm{d}}$ & $0.04^{\mathrm{c}}$ \\
\hline & Second & $2.73^{\mathrm{cd}}$ & $11.93^{b c}$ & $58.87^{b}$ & $1.59^{\mathrm{cd}}$ & $1.16^{\mathrm{bc}}$ & $0.49 b c$ & $0.33^{\mathrm{cd}}$ & $0.22^{\mathrm{ab}}$ \\
\hline & Third & $2.94^{\mathrm{ab}}$ & $16.61^{\mathrm{ab}}$ & $67.10^{\mathrm{ab}}$ & $3.35^{\mathrm{abc}}$ & $1.60^{\mathrm{ab}}$ & $115 \mathrm{ab}$ & $0.57^{b c}$ & $0.21^{\mathrm{ab}}$ \\
\hline
\end{tabular}

* $\mathrm{L}=$ large size $* \mathrm{M}=$ medium size $* \mathrm{~S}=$ small size

Table (8): Manifested that combination treatments among seed size, growth media and periods were affected significantly at level of significance 0.01 on the studied characters. The highest values of (shoot diameter, shoot length, shoot fresh weight, root fresh weight, shoot dry weight, and root dry weight) were $(4.11,26.50$, $10.71,4.25,3.88$, and 1.84) which recorded from combination treatment of (third period, large seed size and media 3) respectively, and the highest values of root length and quality index (101.33 and 0.69) were noticed from treatment combinations (medium seed size- third period- media1) and (medium seed sizesecond period- media1) respectively. While the lowest values of (shoot diameter, root length, shoot fresh weight, and root fresh weight) were $(1.18,26.33,0.37$, and 0.33 ) were obtained from treatment combinations ( small seed size, first period, and media1), on the other hand the lowest values of shoot dry weight, root dry weight and shoot length were $(0.04,0.01$ and 4.67$)$ recorded from treatment combinations(small seed size, first period, and media4) and (small seed size, second period, and media3) respectively. There interaction created different conditions for plant growth which were affected on the studied characters (Darwesh, 2007). 
Table (8): Interaction effect of different period, seed size and growing media on some characteristics of Pinus brutia Ten.seedling.

\begin{tabular}{|c|c|c|c|c|c|c|c|c|c|c|}
\hline \multirow[t]{2}{*}{$\begin{array}{l}\text { Large } \\
\text { Seed } \\
\text { Size }\end{array}$} & \multirow[t]{2}{*}{$\begin{array}{l}\text { Peri } \\
\text { od }\end{array}$} & \multirow[t]{2}{*}{$\begin{array}{c}\text { medi } \\
\text { a }\end{array}$} & \multirow[t]{2}{*}{$\begin{array}{l}\text { shoot } \\
\text { diameter } \\
(\mathrm{mm})\end{array}$} & $\begin{array}{l}\text { shoot } \\
\text { length }\end{array}$ & $\begin{array}{c}\text { root } \\
\text { length }\end{array}$ & $\begin{array}{c}\text { shoot } \\
\text { fresh } \\
\text { weight }\end{array}$ & $\begin{array}{c}\text { root } \\
\text { fresh } \\
\text { weight }\end{array}$ & $\begin{array}{l}\text { shoot dry } \\
\text { weight }\end{array}$ & $\begin{array}{c}\text { root } \\
\text { dry } \\
\text { weight }\end{array}$ & \multirow[t]{2}{*}{$\begin{array}{c}\text { Qualit } \\
\text { y } \\
\text { index }\end{array}$} \\
\hline & & & & \multicolumn{2}{|c|}{$(\mathrm{cm})$} & \multicolumn{4}{|c|}{ (g) } & \\
\hline \multirow{15}{*}{$\mathrm{L}$} & \multirow{5}{*}{$\begin{array}{c}\text { Firs } \\
\mathrm{t}\end{array}$} & M1 & $1.93^{\mathrm{g}-\mathrm{o}}$ & $6.53^{\mathrm{h}-\mathrm{k}}$ & $35.73^{d-g}$ & $0.70^{\mathrm{g}}$ & $0.87^{\text {efg }}$ & $0.21^{\mathrm{i}}$ & $0.19^{\mathrm{gh}}$ & $0.09^{\mathrm{fg}}$ \\
\hline & & M2 & $1.89^{\mathrm{g}-\mathrm{o}}$ & $7.50^{\mathrm{g}-\mathrm{k}}$ & $42.03^{\mathrm{c}-\mathrm{g}}$ & $0.78^{\mathrm{g}}$ & $1.06^{\mathrm{efg}}$ & $0.25^{\mathrm{i}}$ & $0.19^{\text {gh }}$ & $0.08^{\mathrm{fg}}$ \\
\hline & & M3 & $2.63^{\mathrm{e}-1}$ & $9.77^{\mathrm{d}-\mathrm{k}}$ & $33.33^{\mathrm{fg}}$ & $1.19^{\mathrm{fg}}$ & $1.15^{\mathrm{d}-\mathrm{g}}$ & $0.55^{\mathrm{hi}}$ & $0.19^{\text {gh }}$ & $0.01^{\mathrm{d}-\mathrm{g}}$ \\
\hline & & M4 & $1.37^{\mathrm{no}}$ & $7.67^{\mathrm{g}-\mathrm{k}}$ & $54^{b-g}$ & $0.73^{\mathrm{g}}$ & $1.27^{\mathrm{c}-\mathrm{g}}$ & $0.19^{i}$ & $0.17^{\text {gh }}$ & $0.04^{\mathrm{g}}$ \\
\hline & & M5 & $1.75^{1-\mathrm{o}}$ & $8.33^{\mathrm{f}-\mathrm{k}}$ & $44^{\mathrm{c}-\mathrm{g}}$ & $1.02^{\mathrm{fg}}$ & $1.23^{\mathrm{c}-\mathrm{g}}$ & $0.20^{i}$ & $0.15^{\mathrm{gh}}$ & $0.05^{\mathrm{g}}$ \\
\hline & \multirow{5}{*}{$\begin{array}{l}\text { Sec } \\
\text { ond }\end{array}$} & M1 & $2.46^{\mathrm{e}-\mathrm{m}}$ & $10.80^{\mathrm{c}-\mathrm{k}}$ & $40.33^{c-g}$ & $1.16^{\mathrm{fg}}$ & $0.75^{\mathrm{fg}}$ & $0.29^{i}$ & $0.28^{\mathrm{fgh}}$ & $0.67^{\mathrm{a}}$ \\
\hline & & M2 & $2.66^{\mathrm{e}-1}$ & $9.33^{\mathrm{d}-\mathrm{k}}$ & $62.33^{a-g}$ & $1.02^{\mathrm{fg}}$ & $1.17^{\mathrm{d}-\mathrm{g}}$ & $0.35^{\mathrm{i}}$ & $0.31^{\text {fgh }}$ & $0.14^{\mathrm{d}-\mathrm{g}}$ \\
\hline & & M3 & $3.60^{\mathrm{a}-\mathrm{d}}$ & $17.37^{b}$ & $50^{b-g}$ & $4.60^{\text {cde }}$ & $2.60^{b-f}$ & $1.37^{\mathrm{e}-\mathrm{h}}$ & $0.77^{b-f}$ & $0.33^{\mathrm{b}-\mathrm{g}}$ \\
\hline & & M4 & $3.11^{\mathrm{b}-\mathrm{h}}$ & $12.33^{\mathrm{b}-\mathrm{h}}$ & $64.67^{\mathrm{a}-\mathrm{g}}$ & $1.86^{\mathrm{efg}}$ & $1.73^{\mathrm{b}-\mathrm{g}}$ & $0.63^{\text {ghi }}$ & $0.52^{\mathrm{e}-\mathrm{h}}$ & $0.22^{\mathrm{c}-\mathrm{g}}$ \\
\hline & & M5 & $3^{c-h}$ & $14.83^{\mathrm{bc}} \mathrm{d}$ & $70^{\mathrm{a}-\mathrm{f}}$ & $3.03^{\mathrm{d}-\mathrm{g}}$ & $2.35^{\mathrm{b}-\mathrm{f}}$ & $0.97^{\mathrm{fi}}$ & $0.78^{\mathrm{b}-\mathrm{f}}$ & $0.28^{\mathrm{c}-\mathrm{g}}$ \\
\hline & \multirow{5}{*}{$\begin{array}{l}\text { Thi } \\
\text { rd }\end{array}$} & M1 & $3.25^{\mathrm{a}-\mathrm{e}}$ & $11^{\mathrm{c}-\mathrm{k}}$ & $56.33^{\mathrm{b}-\mathrm{g}}$ & $1.89^{\mathrm{efg}}$ & $1.19^{\mathrm{c}-\mathrm{g}}$ & $0.60^{\mathrm{hi}}$ & $0.49^{\mathrm{e}-\mathrm{h}}$ & $0.33^{c-g}$ \\
\hline & & M2 & $2.55^{\mathrm{e}-\mathrm{m}}$ & $12^{\mathrm{b}-\mathrm{i}}$ & $58.50^{\mathrm{b}-\mathrm{g}}$ & $1.33^{\mathrm{fg}}$ & $1.24^{\mathrm{c}-\mathrm{g}}$ & $0.53^{\text {hi }}$ & $0.38^{\mathrm{e}-\mathrm{h}}$ & $0.20^{\mathrm{d}-\mathrm{g}}$ \\
\hline & & M3 & $4.11^{\mathrm{a}}$ & $26.50^{\mathrm{a}}$ & $56.67^{\mathrm{b}-\mathrm{g}}$ & $10.71^{\mathrm{a}}$ & $4.25^{\mathrm{a}}$ & $3.88^{\mathrm{a}}$ & $1.84^{\mathrm{a}}$ & $0.67^{\mathrm{a}}$ \\
\hline & & M4 & $3.15^{b-g}$ & $16.17^{\mathrm{bc}}$ & $59.67^{b-g}$ & $3.19^{\mathrm{d}-\mathrm{g}}$ & $2.07^{\mathrm{b}-\mathrm{g}}$ & $1.58^{\mathrm{d}-\mathrm{g}}$ & $0.84^{\mathrm{b}-\mathrm{e}}$ & $0.30^{\mathrm{b}-\mathrm{g}}$ \\
\hline & & M5 & $3.67^{\mathrm{abc}}$ & $23.67^{\mathrm{a}}$ & $77^{\mathrm{a}-\mathrm{d}}$ & $7.25^{\mathrm{bc}}$ & $2.99^{\mathrm{ab}}$ & $2.49^{\text {bcd }}$ & $1.09^{\mathrm{bcd}}$ & $0.39^{\text {bcd }}$ \\
\hline \multirow[t]{2}{*}{$\begin{array}{c}\text { Medium } \\
\text { Seed } \\
\text { size }\end{array}$} & \multirow[t]{2}{*}{$\begin{array}{l}\text { Peri } \\
\text { od }\end{array}$} & \multirow[t]{2}{*}{$\begin{array}{c}\text { medi } \\
\text { a }\end{array}$} & \multirow[t]{2}{*}{$\begin{array}{c}\text { shoot } \\
\text { diameter } \\
(\mathrm{mm})\end{array}$} & $\begin{array}{l}\text { shoot } \\
\text { length }\end{array}$ & $\begin{array}{l}\text { root } \\
\text { length }\end{array}$ & $\begin{array}{l}\text { shoot } \\
\text { fresh } \\
\text { weight }\end{array}$ & $\begin{array}{c}\text { root } \\
\text { fresh } \\
\text { weight }\end{array}$ & $\begin{array}{l}\text { shoot dry } \\
\text { weight }\end{array}$ & $\begin{array}{c}\text { root } \\
\text { dry } \\
\text { weight }\end{array}$ & \multirow[t]{2}{*}{$\begin{array}{c}\text { Qualit } \\
\text { y } \\
\text { index }\end{array}$} \\
\hline & & & & \multicolumn{2}{|c|}{$(\mathrm{cm})$} & \multicolumn{4}{|c|}{ (g) } & \\
\hline \multirow{15}{*}{ M } & \multirow{5}{*}{$\begin{array}{c}\text { Firs } \\
\mathrm{t}\end{array}$} & M1 & $1.64^{\mathrm{mno}}$ & $6.10^{\mathrm{ijk}}$ & $40.33^{\mathrm{c}-\mathrm{g}}$ & $0.57^{\mathrm{g}}$ & $0.85^{\mathrm{efg}}$ & $0.17^{i}$ & $0.16^{\mathrm{gh}}$ & $0.07^{\mathrm{fg}}$ \\
\hline & & M2 & $1.80^{\mathrm{k}-\mathrm{o}}$ & $6.80^{h-k}$ & $34.27^{\text {efg }}$ & $0.71^{\mathrm{g}}$ & $0.75^{\mathrm{fg}}$ & $0.19^{i}$ & $0.11^{\mathrm{gh}}$ & $0.05^{\mathrm{g}}$ \\
\hline & & M3 & $2.47^{\mathrm{e}-\mathrm{m}}$ & $8.37^{\mathrm{f}-\mathrm{k}}$ & $34^{\mathrm{fg}}$ & $1.03^{\mathrm{fg}}$ & $1.08^{\mathrm{d}-\mathrm{g}}$ & $0.21^{\mathrm{i}}$ & $0.15^{\mathrm{gh}}$ & $0.06^{\mathrm{g}}$ \\
\hline & & M4 & $1.65^{\mathrm{mno}}$ & $7^{\mathrm{h}-\mathrm{k}}$ & $55.67^{\mathrm{b}-\mathrm{g}}$ & $0.79^{\mathrm{g}}$ & $1.47^{\mathrm{b}-\mathrm{g}}$ & $0.15^{\mathrm{i}}$ & $0.17^{\text {gh }}$ & $0.07^{\mathrm{fg}}$ \\
\hline & & M5 & $1.67^{\mathrm{mno}}$ & $7.47^{\mathrm{g}-\mathrm{k}}$ & $42.33^{\mathrm{c}-\mathrm{g}}$ & $0.72^{\mathrm{g}}$ & $1.08^{\mathrm{d}-\mathrm{g}}$ & $0.17^{i}$ & $0.12^{\text {gh }}$ & $0.05^{\mathrm{g}}$ \\
\hline & \multirow{5}{*}{$\begin{array}{l}\text { Sec } \\
\text { ond }\end{array}$} & M1 & $2.56^{\mathrm{e}-\mathrm{m}}$ & $9.70^{d-k}$ & $53^{b-g}$ & $1.09^{\mathrm{fg}}$ & $0.98^{\text {efg }}$ & $0.39^{\mathrm{hi}}$ & $0.37^{\mathrm{e}-\mathrm{h}}$ & $0.69^{\mathrm{a}}$ \\
\hline & & M2 & $2.90^{\mathrm{c}-\mathrm{i}}$ & $9.17^{d-k}$ & $65.67^{\mathrm{a}-\mathrm{g}}$ & $1.04^{\mathrm{fg}}$ & $1.03^{\text {efg }}$ & $0.36^{i}$ & $0.38^{\mathrm{e}-\mathrm{h}}$ & $0.18^{\mathrm{d}-\mathrm{g}}$ \\
\hline & & M3 & $3.04^{b-h}$ & $16.67^{\mathrm{bc}}$ & $66.67^{\mathrm{a}-\mathrm{g}}$ & $3.87^{\mathrm{def}}$ & $2.35^{\mathrm{b}-\mathrm{f}}$ & $1.02^{f-i}$ & $0.61^{\mathrm{d}-\mathrm{h}}$ & $0.22^{\mathrm{c}-\mathrm{g}}$ \\
\hline & & M4 & $2.55^{\mathrm{e}-\mathrm{m}}$ & $12.33^{\mathrm{b}-\mathrm{h}}$ & $61^{a-g}$ & $1.16^{\mathrm{fg}}$ & $1.03^{\text {efg }}$ & $0.37^{\mathrm{hi}}$ & $0.29^{\text {fgh }}$ & $0.11^{\mathrm{d}-\mathrm{g}}$ \\
\hline & & M5 & $2.55^{\mathrm{e}-\mathrm{m}}$ & $17.67^{b}$ & $66.67^{\mathrm{a}-\mathrm{g}}$ & $3.22^{\mathrm{d}-\mathrm{g}}$ & $1.86^{\mathrm{b}-\mathrm{g}}$ & $0.92^{f-i}$ & $0.52^{\mathrm{e}-\mathrm{h}}$ & $0.17^{\mathrm{d}-\mathrm{g}}$ \\
\hline & \multirow{5}{*}{$\begin{array}{l}\text { Thi } \\
\text { rd }\end{array}$} & M1 & $2.73^{\mathrm{d}-\mathrm{k}}$ & $11.83^{b-j}$ & $101.33^{\mathrm{a}}$ & $1.97^{\text {efg }}$ & $1.21^{\mathrm{c}-\mathrm{g}}$ & $0.76^{\text {ghi }}$ & $0.50^{\mathrm{e}-\mathrm{h}}$ & $0.21^{\mathrm{c}-\mathrm{g}}$ \\
\hline & & M2 & $2.54^{\mathrm{e}-\mathrm{m}}$ & $9.23^{\mathrm{d}-\mathrm{k}}$ & $62.33^{a-g}$ & $0.95^{\mathrm{fg}}$ & $0.92^{\text {efg }}$ & $0.33^{i}$ & $0.39^{\mathrm{e}-\mathrm{h}}$ & $0.16^{\mathrm{d}-\mathrm{g}}$ \\
\hline & & M3 & $3.92^{\mathrm{ab}}$ & $24.67^{\mathrm{a}}$ & $60^{\mathrm{b}-\mathrm{g}}$ & $9.06^{\mathrm{ab}}$ & $3.04^{\mathrm{ab}}$ & $3.19^{\mathrm{ab}}$ & $1.23^{\mathrm{e}}$ & $0.50^{\mathrm{abc}}$ \\
\hline & & M4 & $2.80^{c-j}$ & $14.33^{\mathrm{b}-\mathrm{f}}$ & $80^{\mathrm{abc}}$ & $2.61^{\text {efg }}$ & $1.86^{\mathrm{b}-\mathrm{g}}$ & $0.99^{\mathrm{f}-\mathrm{i}}$ & $0.64^{\mathrm{c}-\mathrm{g}}$ & $0.24^{\mathrm{c}-\mathrm{g}}$ \\
\hline & & M5 & $3.59^{\mathrm{a}-\mathrm{d}}$ & $25.27^{\mathrm{a}}$ & $86.67^{\mathrm{ab}}$ & $6.75^{\mathrm{bc}}$ & $2.91^{\mathrm{abc}}$ & $2.26^{\text {cde }}$ & $1.10^{\mathrm{bc}}$ & $0.37^{b-f}$ \\
\hline
\end{tabular}




\begin{tabular}{|c|c|c|c|c|c|c|c|c|c|c|}
\hline \multirow[t]{2}{*}{$\begin{array}{l}\text { Small } \\
\text { Seed } \\
\text { size }\end{array}$} & \multirow[t]{2}{*}{$\begin{array}{c}\text { Peri } \\
\text { od }\end{array}$} & \multirow[t]{2}{*}{$\begin{array}{c}\text { medi } \\
\text { a }\end{array}$} & \multirow[t]{2}{*}{$\begin{array}{c}\text { shoot } \\
\text { diameter } \\
(\mathrm{mm})\end{array}$} & $\begin{array}{l}\text { shoot } \\
\text { length }\end{array}$ & $\begin{array}{c}\text { root } \\
\text { length }\end{array}$ & $\begin{array}{c}\text { shoot } \\
\text { fresh } \\
\text { weight }\end{array}$ & $\begin{array}{c}\text { root } \\
\text { fresh } \\
\text { weight }\end{array}$ & $\begin{array}{l}\text { shoot dry } \\
\text { weight }\end{array}$ & $\begin{array}{c}\text { root } \\
\text { dry } \\
\text { weight }\end{array}$ & \multirow[t]{2}{*}{$\begin{array}{c}\text { Qualit } \\
\text { y } \\
\text { index }\end{array}$} \\
\hline & & & & \multicolumn{2}{|c|}{$(\mathrm{cm})$} & \multicolumn{4}{|c|}{ (g) } & \\
\hline \multirow{15}{*}{$S$} & \multirow{5}{*}{$\begin{array}{c}\text { Firs } \\
\mathrm{t}\end{array}$} & M1 & $1.18^{\circ}$ & $5.23^{k}$ & $26.33^{\mathrm{g}}$ & $0.37^{\mathrm{g}}$ & $0.33^{\mathrm{g}}$ & $0.17^{\mathrm{i}}$ & $0.11^{\mathrm{h}}$ & $0.03^{\mathrm{g}}$ \\
\hline & & M2 & $1.62^{\mathrm{mno}}$ & $5.83^{\mathrm{jk}}$ & $37.73^{\mathrm{fg}}$ & $0.48^{\mathrm{g}}$ & $0.59^{\mathrm{fg}}$ & $0.11^{\mathrm{i}}$ & $0.09^{h}$ & $0.04^{\mathrm{g}}$ \\
\hline & & M3 & $1.89^{\mathrm{g}-\mathrm{o}}$ & $7.80^{\mathrm{g}-\mathrm{k}}$ & $33.33^{\mathrm{fg}}$ & $1.28^{\mathrm{fg}}$ & $1.03^{\mathrm{efg}}$ & $0.20^{\mathrm{i}}$ & $0.17^{\mathrm{gh}}$ & $0.07^{\mathrm{g}}$ \\
\hline & & M4 & $1.64^{\mathrm{mno}}$ & $6.13^{\mathrm{ijk}}$ & $45^{\mathrm{b}-\mathrm{g}}$ & $0.56^{\mathrm{g}}$ & $0.79^{\mathrm{fg}}$ & $0.04^{i}$ & $0.01^{\mathrm{h}}$ & $0.03^{\mathrm{g}}$ \\
\hline & & M5 & $1.43^{\mathrm{no}}$ & $7.37^{\mathrm{h}-\mathrm{k}}$ & $36.67^{d-g}$ & $0.48^{\mathrm{g}}$ & $0.59^{\mathrm{fg}}$ & $0.08^{i}$ & $0.11^{\mathrm{h}}$ & $0.03^{\mathrm{g}}$ \\
\hline & \multirow{5}{*}{$\begin{array}{l}\text { Sec } \\
\text { ond }\end{array}$} & M1 & $2.17^{\mathrm{h}-\mathrm{n}}$ & $8.50^{\mathrm{e}-\mathrm{k}}$ & $56^{\mathrm{b}-\mathrm{g}}$ & $0.69^{g}$ & $0.75^{\mathrm{fg}}$ & $0.26^{\mathrm{i}}$ & $0.21^{\mathrm{gh}}$ & $0.56^{\mathrm{ab}}$ \\
\hline & & M2 & $2.25^{\mathrm{f}-\mathrm{n}}$ & $8.50^{\mathrm{e}-\mathrm{k}}$ & $55^{\mathrm{b}-\mathrm{g}}$ & $0.97^{\mathrm{fg}}$ & $1.04^{\mathrm{efg}}$ & $0.32^{\mathrm{i}}$ & $0.28^{\mathrm{fgh}}$ & $0.11^{\mathrm{d}-\mathrm{g}}$ \\
\hline & & M3 & $3.09^{b-h}$ & $4.67^{\mathrm{bcd}}$ & $54.33^{\mathrm{b}-\mathrm{g}}$ & $3.37^{\mathrm{d}-\mathrm{g}}$ & $2.03^{b-g}$ & $0.99^{f-i}$ & $0.57^{\mathrm{e}-\mathrm{h}}$ & $0.23^{\mathrm{c}-\mathrm{g}}$ \\
\hline & & M4 & $2.39^{\mathrm{e}-\mathrm{m}}$ & $14.50^{\mathrm{b}-\mathrm{d}}$ & $70.67^{\mathrm{a}-\mathrm{f}}$ & $1.54^{\mathrm{fg}}$ & $1.20^{\mathrm{c}-\mathrm{g}}$ & $0.50^{\mathrm{hi}}$ & $0.37^{\mathrm{e}-\mathrm{h}}$ & $0.12^{\mathrm{d}-\mathrm{g}}$ \\
\hline & & M5 & $1.97^{\mathrm{i}-\mathrm{o}}$ & $13.50^{b-g}$ & $53.33^{\mathrm{b}-\mathrm{g}}$ & $1.39^{\mathrm{fg}}$ & $0.79^{\mathrm{fg}}$ & $0.40^{\mathrm{hi}}$ & $0.26^{\mathrm{gh}}$ & $0.08^{\mathrm{fg}}$ \\
\hline & \multirow{5}{*}{$\begin{array}{l}\text { Thi } \\
\text { rd }\end{array}$} & M1 & $2.98^{\mathrm{c}-\mathrm{h}}$ & $8.53^{\mathrm{e}-\mathrm{k}}$ & $73^{a-f}$ & $0.80^{\mathrm{g}}$ & $0.75^{\mathrm{fg}}$ & $0.35^{\mathrm{i}}$ & $0.22^{\mathrm{gh}}$ & $0.13^{\mathrm{d}-\mathrm{g}}$ \\
\hline & & M2 & $2.21^{g-n}$ & $9.20^{\mathrm{d}-\mathrm{k}}$ & $56.17^{\mathrm{b}-\mathrm{g}}$ & $0.76^{\mathrm{g}}$ & $0.71^{\mathrm{fg}}$ & $0.26^{i}$ & $0.24^{\text {gh }}$ & $0.09^{\text {efg }}$ \\
\hline & & M3 & $3.33^{\mathrm{a}-\mathrm{e}}$ & $25.67^{\mathrm{a}}$ & $76.67^{\mathrm{a}-\mathrm{e}}$ & $7.13^{b c}$ & $2.81^{\mathrm{a}-\mathrm{d}}$ & $2.52^{\mathrm{bc}}$ & $1.09^{\mathrm{bcd}}$ & $0.39^{\mathrm{b}-\mathrm{e}}$ \\
\hline & & M4 & $3.02^{b-h}$ & $13.97^{b-f}$ & $71^{\mathrm{a}-\mathrm{f}}$ & $2.49^{\mathrm{efg}}$ & $1.78^{\mathrm{b}-\mathrm{g}}$ & $0.86^{\mathrm{f}-\mathrm{i}}$ & $0.53^{\mathrm{e}-\mathrm{h}}$ & $0.20^{\mathrm{d}-\mathrm{g}}$ \\
\hline & & M5 & $3.18^{b-f}$ & $25.67^{\mathrm{a}}$ & $58.67^{\mathrm{b}-\mathrm{g}}$ & $5.57^{\mathrm{cd}}$ & $1.99^{\mathrm{b}-\mathrm{g}}$ & $1.77^{\mathrm{c}-\mathrm{f}}$ & $0.78^{b-f}$ & $0.25^{\mathrm{c}-\mathrm{g}}$ \\
\hline
\end{tabular}

\section{CONCLUSION}

From the present investigation it can be concluded that germination, survival, growth of Pinus brutia Ten. were enhanced by using the large seed while the germination percentage significantly declined with reduction in seed size. The growth media were affected significantly on most of the studied characters .The highest values of studied characteristics of seedlings were recorded from media 3 (field soil+ compost). This indicates that such mixture can be regarded as a suitable growth media component, depending on the amount of compost, plant growing requirement, and the specific chemical characteristics desired in the growth media. The combination treatments among seed size, growth media and periods were affected significantly on the studied characters, so we recommend that our results should be taken into consideration by forest nursery in Kurdistan because it could enhance and may advance the quality and quantity of seedling production of Pinus brutia Ten. 


\section{REFERENCES}

Ali, N.S. (2015). Temporal variation of the nutrients content of the forest trees and soils at Hijran and Zawita sites, MSc. Thesis. Univ. of Salahaddin. College of Agriculture.

Ahmadloo, F., Tabari, M., Yousef zadeh H., Kooch , Y., and Rahmani, A. (2012). Effects of soil nutrient on seedling performance of arizona cypress and medite cypress. Annals of Biological Research, 3 (3):1369-1380

Aklibasinda,M.; Tunc, T. Bulut, Y. and Sahin, U. (2011). Effect of different growth media on Scotch pine (Pinus sylvestris) production. The Journal of Animal \& Plant Sciences, 21(3): 535-541 ,ISSN: 1018-7081

Aldrete, A. and Mexal, J. G.(2005). Sowing Depth, Media, and Seed Size Interact To Influence Emergence of Three Pine Species. Tree Planter's Notes, Vol. 51, No.1.

Al-Rawi, K. M. and Khalaf-Allah, A. M. (2000). Design and analysis for Agricultural experiments. Directorate of book house of publishing and pressing. University of Mosul. Iraq.

Arjunan, M.C., Antony, K.A. and Ponnammal, N.R. (1994). Effect of seed size on germination, viability and seedling biomass in Pongamia pinnata Pierre, Van Vigyan, 32, 23-28.

Cicek, E. and Tilki, F. (2007). Seed size effects on germination, survival and seedling growth of Castanea sativa Mill. Journal of Biological Sciences 7(2):438441.

Darwesh, D.A. (2007). Role of Supplemental Irrigation and Fertilizer Treatments on Yield and Nutrients Balance in Wheat by Using Modified DRIS, PhD. Thesis. Univ.of Salahaddin, College of Agriculture.

Durgapal, A., Pandey, A. and Palni, L.M.( 2002). The use of rhizosphere soil for improved establishment of conifers at nursery stage for application in plantation programmes. J. Sustainable For., 15: 57-73.

Ellis, R.H. (1992). "Seed and seedling vigor in relation to crop growth and yield." Plant Growth Regulation, 11, 249-255.

Foster, S. A. (1986). On the adaptive value of large seeds for tropical moist forest trees: a review and synthesis. Botanical Review 52: 260-299.

Himanen, K., Pekka, H., Tiina, Y. and Markku, N. (2016). Intracone variation explains most of the variance in Picea abies seed weight: implications for seed sorting, Can. J. For. Res. 46: 470-477.

Indira, E.P. Chand Basha S. and Chacko, K.C. (2000). Effect of seed size grading on germination and growth of Teak (Tectona grandis) seedlings, J. Trop. For. Sci., $12(1), 21-27$.

Ismail, O. M. and M. Kardoush (2011). The impact of some nutrient substances on germination and growth seedling of Pistacia vera L. Australian J. Basic and Applied Sci. 5(5): 115-120. 
Lusk, C.H. (1995). Seed size, establishment sites and species co-existence in a Chilean rain forest, Jour. Vegetation Sci., 6, 249-256 .

Mathur, S. P., and Voisin, B. (1996). The use of compost as greenhouse growth media. Final report, Ministry of Environment and Energy, Ontario.

Mazer, S. J. (1989). Ecological, taxonomic and life history correlates of seed mass among Indiana dune angiosperms. Ecological Monographs 59: 153-175.

Olle, M. and Akvile, V. (2013). The effects of light-emitting diode lighting on greenhouse plant growth and quality. Agricultural and food science. 22:223-234.

Parker ,W.C. , Noland ,T.L. and Morneault ,A.E. (2004). Effect of seed mass on early seedling growth of five eastern white pine (Pinus strobus L.) families under contrasting light environments. Can. J. Bot. 82: 1645-1655.

SAS, (2005). Statistical analysis system institute, Cary NC, USA.

Şahin, Ü., A. Hanay, and Anapal, Ö. (1997). A study on the use of pumice in soilless culture in greenhouses. Proc. 1st Isparta Pumice Symposium, Isparta, 133139.

Salisbury, J. W. and Graham, R. H. (1974). Meteorite Spectra and Weathering. Journal of geophysical research. vol. 79, NO. 29.

Seiwa, K. and Kikuzawa, K. (1991). Phenology of tree seedlings in relation to seed size. Canadian Journal of Botany 69: 532-538.

Sharma, K.K. and Sood, O.P. (1990). Germination behavior of seed of Leucaena leucocephala, as influenced by various treatments and seed sizes, Van Vigyan, 28 (3),99-105.

Singh, V. and Shah, V.K. (1992). Effect of seed weight on germination and seedling growth in Silver fir (Abies pindrow ), Van Vigyan, 30, 104-106 .

Tabatabaii, M. and Ghasriani, F. (1993). Natural resources of Kurdistan. Jahade daneshgahi Publication.767 p.

Thompson, B.E. (1984). Establishing a vigorous nursery crop: bed preparation, seed sowing and early seed growth. In: Forest nursery manual- production of bareroot seedlings (M. L. Duryea and T. D., Landis, eds.). Forest Research Laboratory, Oregon State University. Martines Nijhoff/Dr.W. Junk Publishers, 41$49 \mathrm{p}$.

Vilela, A. E. and Ravetta, D. A. (2001). The effect of seed scarification and soil media on germination, growth, storage and survival of seedlings of five species of Prosopis L. (mimosaceae). Arid Environments. 48 : 171-184. 